\title{
PedsQL-4 Total Scale Score
}

National Cancer Institute

\section{Source}

National Cancer Institute. PedsQL-4 Total Scale Score. NCI Thesaurus. Code C121531.

The mean value computed as the sum of all individual items over the number of items answered on all the Scales. 